\title{
Lentivirus-mediated shRNA targeting decoy receptor 3 (DcR3) inhibits proliferation and augments apoptosis in pancreatic cancer Capan-1 cells
}

\author{
Yingchao Zhang ${ }^{1}$, Azhar Rasul ${ }^{2}$, Xiaomeng Li $^{2}$, Mahadev Malhi ${ }^{2}$, Xuedong Fang ${ }^{1}$, Lijuan \\ Wang $^{3}$ and Yu Wang ${ }^{1 *}$ \\ ${ }^{1}$ Department of General Surgery, The Second Hospital of Jilin University, Changchun, 130041, P.R. China. \\ ${ }^{2}$ Membrane Channel Research Laboratory, Northeast Normal University, Changchun, 130024, P.R. China. \\ ${ }^{3}$ Department of General Surgery, Mining Group General hospital of Liaoyuan, Jilin Province Liaoyuan, 136201, \\ P.R. China.
}

Accepted 19 November, 2012

\begin{abstract}
Pancreatic cancer is one of the most dismal malignancies with the actual 5-year survival of only 10 to $20 \%$. Decoy receptor 3 (DcR3) is highly expressed in various cancer cells and plays a significant role in immune suppression and tumor progression. However, how DcR3 expression is modulated in pancreatic cancer cells is enigmatic. The aim of this study was to characterize the expression of DcR3 in pancreatic carcinoma and to evaluate the role of DcR3 in cell proliferation and apoptosis, which may lead to the development of novel treatments for this disease. In the present study, we examined five cell lines including three cell lines from pancreatic cancer (SW1990, Capcan-1 and PANC-1) and two cell lines from colon cancer (HCT116 and RKO) for DcR3 expression by reverse transcriptase-polymerase chain reaction (RT-PCR) and quantitative real time PCR. 3'-(4, 5 dimethyl-thiazol-2-yl)-2, 5-diphenyl tetrazolium bromide (MTT) assay and flow cytometric analysis were used to measure cell proliferation and apoptosis, respectively in the human pancreatic cancer Capan-1 cells. The human pancreatic cancer Capan-1 cell line was first infected with lentivirus-mediated shRNA and then analyzed by realtime PCR, and the results were further confirmed by Western blotting. DcR3 protein in our experimental results showed significant expression among the pancreatic cancer cell lines and their decreased expression by lentivirus-mediated shRNA resulted in the inhibition of cell proliferation and augmentation of apoptosis. In conclusion, these findings suggest that lentivirus-mediated gene therapy targeting DcR3 is a potential and attractive strategy for the treatment of pancreatic cancer.
\end{abstract}

Key words: Pancreatic cancer, decoy receptor 3 (DcR3), Capcan-1, shRNA.

\section{INTRODUCTION}

Pancreatic cancer is the fourth leading cause of cancerrelated deaths in United States (Jemal et al., 2009). Current treatment of pancreatic cancer includes surgery, chemotherapy, and radiotherapy, but these therapies are unsatisfactory, time consuming and expensive. Surgical abscission remains the only option for long term survival of patients.

Even after curative resection, the actual 5-year survival

*Corresponding author. E-mail: wangyu720529@yahoo.com.cn. is only 10 to $20 \%$ (Awasthi et al., 2011; Yip-Schneider et al., 2005). Therefore, new options for treatments of pancreatic cancer are desperately needed. Gene therapy provides a new concept and it may become the new emerging direction for the development of treatment method for pancreatic cancer.

Decoy receptor 3 (DcR3), also known as TR6 or M68, is one of the members of tumor necrosis factor receptor (TNFR) superfamily. DcR3 mRNA (full-length $1.2 \mathrm{~kb}$ ), its coding gene M68 located in human chromosome 20q13.3, processing mature the DcR3 271 amino acids, the molecular weight of about $35 \mathrm{KD}$. DcR3 cDNA encodes a 
300-aa protein containing the four tandem cysteine-rich repeats characteristic of the TNFR superfamily and lacking a transmembrane sequence (Pitti et al., 1998). DcR3 can bind with the Fas receptor, LIGHT and TLIA combination, regulate the process of tumor cell apoptosis. Thus, DcR3 is a potential target for treatment of cancer (Shen et al., 2005; Wu et al., 2003). Previous studies revealed that DcR3 is highly expressed in esophageal cancer, stomach cancer, colon tumors, but little is known about the expression of DcR3 in pancreatic cancer cells. Therefore, the purpose of this study was to characterize the expression of DcR3 in pancreatic carcinoma and to evaluate the role of DcR3 in cell proliferation and apoptosis in pancreatic cancer cells. In this study, we examined 5 cell lines including three cell lines from pancreatic cancer (SW1990, Capcan-1 and PANC-1) and two cell lines from colon cancer (HCT116 and RKO) for DcR3 expression by reverse transcriptase-polymerase chain reaction (RT-PCR) and quantitative real time PCR. Our results demonstrated that the DcR3 protein was significantly expressed among the pancreatic cancer cell lines and their decreased expression resulted in the inhibition of cell proliferation and augmentation of apoptosis.

\section{MATERIALS AND METHODS}

\section{Chemicals and reagents}

Cell culture medium reagents and 3'-(4, 5 dimethyl-thiazol-2-yl)-2, 5-diphenyl tetrazolium bromide (MTT), propidium iodide (PI), and dimethyl sulfoxide (DMSO) were purchased from Sigma. Fetal bovine serum (FBS) was purchased from the Hangzhou Sijiqing Biological Engineering Materials Co., Ltd. China. Annexin V-FITC apoptosis detection kit was purchased from Beyotime Institute of Biotechnology Shanghai, China. Mouse anti- $\beta$-actin and anti-rabbit antibodies were purchased from Santa Cruz Biotechnology. Ponceou and cell lysis buffer for Western and IP were purchased from Bio SS Beijing.

\section{Cell culture}

Human pancreatic adenocarcinoma cells SW1990 and Capan-1, human pancreatic epithelioid carcinoma PANC-1, human colon adenocarcinoma cells HCT116 and RKO were obtained from the American Type Culture Collection (Manassas, VA, USA), and cultured in the media recommended by the vendor (RPMI-1640 medium for SW1990 and Capan-1; Dulbecco's modified Eagle's medium for PANC-1 cells; Minimum essential medium Eagle for HCT116 cells; Leibovitz's L-15 medium for RKO cells) supplemented with $10 \%$ (v/v) FBS (Hangzhou Sijiqing Biological Engineering Materials Co., Ltd ,China), $100 \mathrm{U} / \mathrm{ml}$ of penicillin and $100 \mu \mathrm{g} / \mathrm{ml}$ of streptomycin (Biological Industries, Kibbutz Beit Haemek, Israel) at $37^{\circ} \mathrm{C}$ in a humidified atmosphere of $5 \% \mathrm{CO}_{2}$ in air. Cells were seeded in $10 \mathrm{~cm}$ culture dish and allowed to grow to approximately $70 \%$ confluence before experimentation.

\section{Lentivirus transduction and transfection}

Lenti-shRNA-DcR3 and lenti-NC virus plasmids were transfected in Capan-1 cells, as described previously (Fish and Kruithof, 2004).
Capan- 1 cells were transfected with ViraPower packaging mix using $100 \mu$ lipofectamine 2000 reagents according to the manufacturer's instructions. The viral supernatant was harvested $48 \mathrm{~h}$ after transfection, passed through $0.45 \mu \mathrm{m}$ filters and was allowed to concentrate, and the viral titer was determined. The viral supernatant was added into target lenti- 1 cells at multiplicity of infection $(10,100,200)$ with DcR3 and $5 \mu \mathrm{g} / \mathrm{ml}$ polybrene to obtain stably-transfected DcR3-1KD and DcR3-1NC cells.

\section{Real-time RT-PCR}

The isolated RNA subjected to RT-PCR was treated with DNase to avoid amplification of DNA contaminants. The forward and reverse primers are as follows: human DcR3 (GenBank Accession NM_003823.2/NM 032945.2), TCA ATG TGC CAG GCT CTT C and CTG GAA AGC CAC AAA GTC G; GAPDH (GenBank Accession NM 002046), ATT CCA CCC ATG GCA AAT TC and TGG GAT TTC CAT TGA TGA CAA G. Cycle threshold $\left(C_{t}\right)$ method was used to analyze the results. The $C_{t}$ value, which is inversely proportional to the initial template copy number, is the calculated cycle number in which the fluorescence signal emitted is significantly above background levels. The mRNA expression level of target genes was normalized to GAPDH using the $2^{-\Delta \Delta \mathrm{Ct}}$ method, in which $\Delta C_{t}=$ target gene $C_{t}-$ GAPDH $C_{t}$, and $\Delta \Delta C_{t}=\Delta C_{t}$ treatment $-\Delta C_{t}$ control.

\section{Western blotting}

To confirm effect of lenti-shRNA-DcR3 on expression of DcR3 in Capan-1 cells, western blot analysis was performed as described previously (Rasul et al., 2012). Briefly, Capan-1 cells were incubated with lenti-shRNA-DcR3 or lenti-NC for indicated time. Cells were trypsinized, collected in $1.5 \mathrm{ml}$ centrifuge tube and washed with PBS. The cell pellets were resuspended in lysis buffer and were lysed on ice for $30 \mathrm{~min}$. After centrifugation for $15 \mathrm{~min}$, the supernatant fluids were collected and the protein content of the supernatant was measured by the NanoDrop 1000 spectrophotometer (Thermo scientific, USA). The protein lysates were separated by electrophoresis on $12 \%$ sodium dodecyl sulfate (SDS)-polyacrylamide gel and transferred to a polyvinylidene difluoride (PVDF) membrane (Amersham Biosciences, Piscataway, $\mathrm{NJ})$. The membranes were soaked in blocking buffer ( $5 \%$ skimmed milk) for $2 \mathrm{~h}$. To probe for DcR3 and $\beta$-actin, membranes were incubated overnight at $4^{\circ} \mathrm{C}$ with relevant antibodies, followed by appropriate horseradish peroxidase (HRP) conjugated secondary antibodies and enhanced chemiluminescence (ECL) detection.

\section{Cell proliferation assay}

The cytotoxic effects of the shRNA on the cells were determined by MTT assay. Briefly, Capan-1 cells were seeded at a density of $1 \times 10^{4}$ cells per well in 96-well plates and were allowed to grow overnight. Upon exposure of the cells to lenti-shRNA-DcR3 or lentiNC for different time periods, cell viability was measured by MTT assay. After incubation, growth of cells was determined by adding MTT (5 mg/ml in phosphate buffered saline) to each well and incubated for $4 \mathrm{~h}$. After removal of the medium, dimethyl sulfoxide (DMSO) was added to each well and was shaken carefully. The absorbance was read at a wavelength of $490 \mathrm{~nm}$ in a plate reader (ELX 800, BIO-TEK Instruments Inc). The growth curve was plotted against mean values which were calculated using the following equation:

$\mathrm{I} \%=\left[\mathrm{A}_{490}(\right.$ control $)-\mathrm{A}_{490}($ treated $\left.)\right] / \mathrm{A}_{490}($ control $) \times 100$ 

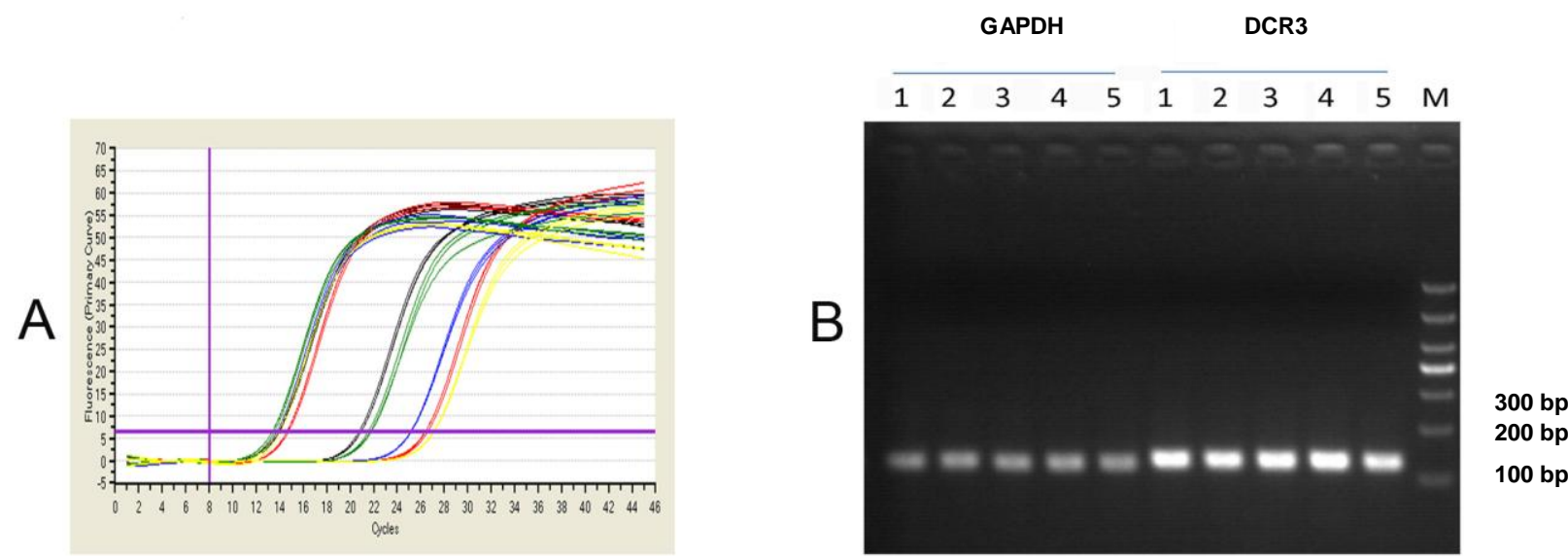

Figure 1. Real-time PCR and reverse transcriptase-PCR analysis of DcR3 expression in different cancer cells: (A) Realtime plot obtained for the quantification of the DcR3 expression in HCT116, PKO, SW1990, Capan-1 and PANC-1 cells (represented by black, red, blue, green, yellow curve respectively). HCT116 cells (black) line SW1990 (blue), in Capan-1 (green) amplification curve CT value, indicating that the curve of the DcR3 gene that corresponds to the initial template, its expression value was higher. (B) DcR3 and GAPDH mRNA expression levels of HCT116 (1), PKO (2), SW1990 (3), Capan-1 (4) and PANC-1(5) cells were assessed by standard RT-PCR. GAPDH served as a loading control.

\section{Flow cytometric determination of apoptosis}

The apoptotic rate of Capan-1 cells was examined by flow cytometry using annexin V-FITC/PI staining as described by us previously (Rasul et al., 2011; Shawi et al., 2011). Briefly, Capan-1 cells were cultured in 6-well plates and allowed to attach overnight. Cells were treated with lenti-shRNA-DcR3 or lenti-NC for different time periods. Cells were then collected, washed and resuspended in PBS. Apoptotic cell death was measured by double staining annexin V-FITC and PI using the Annexin V-FITC apoptosis detection kit (Beyotime Biotechnology Shanghai, China) according to the manufacturer's instructions. Flow cytometric analysis was performed immediately after staining. Data acquisition and analysis were performed by flow cytometry (Beckman Coulter, Inc., Brea, CA) using Cell Quest software.

\section{Statistical analysis of data}

For the statistical analysis of data, comparisons between results from different groups were analyzed with SPSS for Window Version 15.0. Student's $t$-test was employed to determine the statistical significance of the difference between different experimental groups and control group. $\mathrm{P}<0.05$ value was defined as statistically significant. All experiments were repeated at least three times. Data were presented as mean \pm standard deviation (SD).

\section{RESULTS AND DISCUSSION}

In this study, we used real time-PCR and reverse transcriptase-PCR to investigate the presence of DcR3 expression in five cell lines including three cell lines from pancreatic cancer (SW1990, Capcan-1 and PANC-1) and two cell lines from colon cancer (HCT116 and RKO). Human colon cancer cell line HCT116 and RKO were used as the positive control for DcR3 expression. Realtime plot obtained for the quantification of the DcR3 expression in HCT116, PKO, SW1990, Capan-1, PANC-
1 cells, which is represented by black, red, blue, green, yellow curve, respectively for cDNA as a template DCR3 gene primers and GAPDH primers to amplify the target gene and reference gene. HCT116 cells (black), SW1990 (blue), and Capan-1 (green) amplification curve threshold cycle (CT) value lower, indicating that the curve of the DcR3 gene that corresponds to the initial template, its expression values were higher (Figure 1A). Furthermore, DcR3 and GAPDH mRNA expression levels of HCT116 (1), PKO (2), SW1990 (3), Capan-1 (4), and PANC-1(5) cells were assessed by standard RT-PCR. For reverse transcriptase-PCR analysis of DcR3 expression in different cancer cells, results showed that among three, two human pancreatic adenocarcinoma cell lines (SW1990 and Capan-1) have high levels of DcR3 protein, while slightly less level was observed in case of PANC-1 human pancreatic carcinoma cell line (Figure 1B). A previous study (Elnemr et al., 2001) also reported high levels of DcR3 expression in Capan-1 cells. Thus, keeping in view those findings, we selected the Capan-1 cells for the subsequent experiments.

DcR3, also known as TR6 or M68, is one of the members of TNFR superfamily. DcR3 plays a major role in allowing cancer cells to evade attack by the immune system (Elnemr et al., 2001; Li et al., 2007; Pitti et al., 1998). Additional studies have demonstrated that there is a significant correlation between the overexpression of DcR3 and resistance to FasL-mediated apoptosis in cancer cells (Elnemr et al., 2001; Pitti et al., 1998; Shen et al., 2005). Thus, DcR3 is a potential target in treatment of cancer (Shen et al., 2005; Wu et al., 2003). In this study we have constructed five different shRNAs targeting the DcR3 expression in Capan-1 cells.

RNA interference (RNAi) is a powerful tool for studying protein function (Wang et al., 2007; Zhang et al., 2008). 
The effective delivery of siRNA molecules into target cells or tissues is critical for successful RNAi application. Lentiviral vectors can effectively transduce exogenous genes with the long-term expression of transgenes (Fish and Kruithof, 2004) and have a promising future in clinical applications (Thomas et al., 2006; Wang et al., 2007). To determine the role of DcR3 in Capan-1 pancreatic cancer cells, we constructed lentiviral vectors for the delivery of short hairpin RNA, a precursor, into Capan-1 pancreatic cancer cells to suppress the DcR3 gene expression. As shown in Figure $2 \mathrm{~A}$ to $\mathrm{D}$, the number represents (1) negative control group (ddH2O), (2) positive control group (without shRNA fragment with empty vector), (3) Marker (5, 3, 2, 1.5 and $1 \mathrm{~kb}, 750,500,250$, and $100 \mathrm{bp})$, (4 to 8) (A) PSC-1, 2,3,4,5, (B) PSC-1, 2,3,4,5, (C) PSC-1, $2,3,4,5$, and (D) PSC-1, 2,3,4,5. These results suggest that there was no exogenous nucleic acid contamination, which was confirmed by lane number- $1\left(\mathrm{ddH}_{2} \mathrm{O}\right)$ in the system, while lane number-2 is positive group, which confirmed that lane 2 (positive group) for the empty vector clone shRNA (306bp band) is not connected to the target gene. The lane numbers 4 to 8 are positive results confirming for the different RNA interference the target shRNA fragment (that is PSC1/PSC2/positive clones) and virus vector cloning PSC3/PSC4 (PCR fragment size was $343 \mathrm{bp}$ ) in the lane 4 to 8 were selected for the sequencing, and sequencing results confirmed that the target gene (PSC1/PSC2/PSC3/PSC4,) was accurately inserted into the viral vector.

As shown in Figure 2E, (1) negative control group $\left(\mathrm{ddH}_{2} \mathrm{O}\right)$, (2) negative control group (empty eukaryotic expression vector), (3) positive control (GAPDH), (4) Marker, and (5 to 12) TNFRSF6B (1 to 8 transformants). The findings of the present study demonstrated that lane number 1 (negative control group) confirmed that the system has no exogenous nucleic acid contamination, while lane 2 (positive group) for the empty vector (310 bp band) is not connected to the target gene. The lane 3 confirmed connection as a positive control for the GAPDH reference gene carrier. The lanes number 5 to 12 indicated the clear bar with a confirmed carrier is connected to the target gene (the size of the PCR primers for the 1125 bp bands) below the fuzzy bands not connected to the empty vector into the target gene bands. The lanes number 5 to 12 in the PCR positive clones were sent for sequencing and the sequencing results fully consistent with the goal of sequencing, which confirmed that the target gene is accurately connected to the eukaryotic expression vector.

Western blot analysis of three different groups including control group (CON) is not transfected with any plasmid in Capan- 1 cells (empty cells in control group), NC was transfected with expression plasmid and negative control cell group of viral vector plasmid (negative control group). $\mathrm{KD} 1,2,3,4$ contain the target genes for the different interference target sequences of RNAi viral vector plasmid serial number. The corresponding interference plasmid serial were KD1 (4209), KD2 (4210), KD3 (4211), and KD4 (4212). Control group (CON group) and NC group have DcR3 protein expression, indicating that the empty virus vector plasmid DcR3 gene does not significantly inhibited the DcR3 gene expression. KD groups (KD1, KD2 and KD4 groups) significantly decreased DcR3 protein expression as compared to CON and NC group, while KD3 group has no significant influence protein expression. It was noted that out of 4209, 4210, and 4212 targets, 4210 is the most effective shRNA to knockdown the expression of target gene (DcR3) (Figure 2F).

The human pancreatic cancer Capan-1 cell line was infected with shRNA-DcR3 and then analyzed by realtime PCR, and the results were confirmed by Western blotting. Therefore, in the present study, five RNA interference lentiviral vectors were constructed to down regulate the expression of DcR3 in Capan-1 pancreatic cancer cells. Our results demonstrated that these shRNA downregulated the DcR3 expression in Capan-1 cells, then we selected shRNA, which significantly decreased the levels of DcR3 in human pancreatic capan-1 cells (Figure 2A to $\mathrm{E}$ ). The results of the present study were further confirmed by western blot analysis, which demonstrated that shRNA decreased the DcR3 expression in pancreatic cancer cells (Figure $2 \mathrm{~F}$ ).

Morphological changes were observed under microscopy after treating cells with lentivirus-mediated shRNA targeting DCR-3 resulting in the decreased number of cells as compared to the control group and cells became rounded and shrinked, which were polygonal in untreated cells (Figure 3A). Furthermore, our immunofluorescence results demonstrate the successful virus infection of target cells and the infection rate was more than $80 \%$ (Figure 3A). In addition, to analyze the effect of lentivirusmediated shRNA targeting DcR-3, equal amounts of concentrated media from cells were subjected for reverse transcriptase-PCR with the DcR3 primer. Our results illustrate the percentage of relative amount of mRNA in different groups. It was found that Capan-1 cell sampled cDNA as a template to amplify the target gene and reference gene by using DcR3 gene primers. The KD group significantly reduced DcR3 gene expression in comparison to NC group (Figure 3B). To further confirm these findings, Capan-1 cells were exposed to lentivirusmediated shRNA targeting DcR-3 for specified time intervals. Equal amounts of lysate protein were subjected to gel electrophoresis. Expression levels of DcR-3 were monitored in control group (CON), NC, and KD target knockdown in Capan-1 cells by Western blot assay. Our results reveal that DcR3 protein expression was significantly downregulated in KD group as compared to the control group (Figure $3 \mathrm{C}$ ). These results are in consistent with our previous results.

To determine whether lentivirus-mediated shRNA targeting DcR-3 inhibits pancreatic cancer Capan- 1 cell proliferation or not, therefore, we examined its effects on 

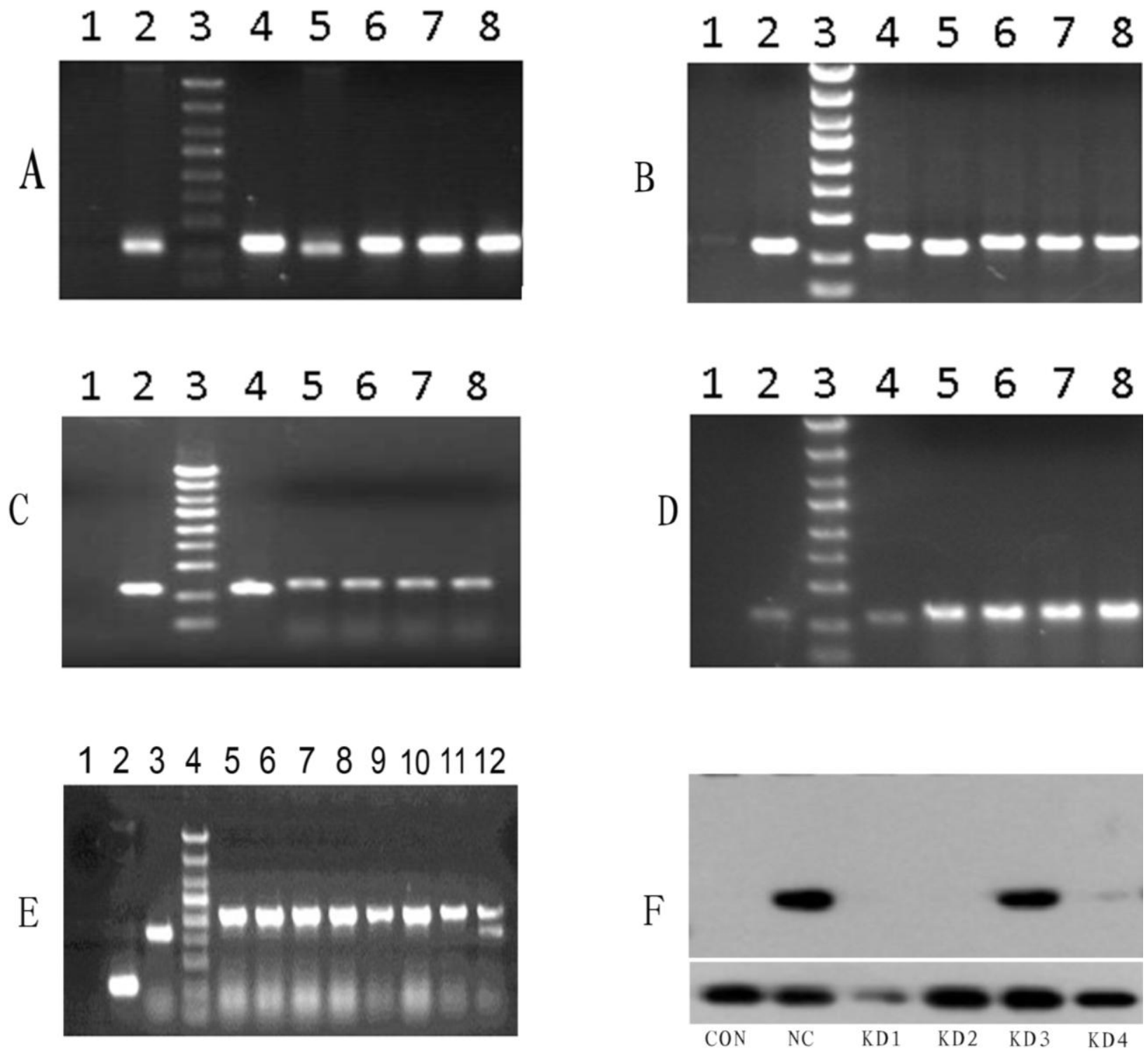

Figure 2. Reverse transcriptase-PCR and immunoblotting assessment of various shRNAs on decoy receptor 3 (DcR3) mRNA and protein expression in Capan-1 pancreatic cancer cells: (A-D) The number represents (1) negative control group (ddH2O), (2) positive control group (without shRNA fragment with empty vector), (3) marker (5, 3, 2, 1.5 and $1 \mathrm{~kb}$ and 750, 500, 250 and 100 bp), (4-8) (A) PSC-1, 2,3,4,5; (B) PSC-1, 2,3,4,5; (C) PSC-1, 2,3,4,5; (D) PSC-1, 2,3,4,5; (E) (1) negative control group (ddH2O), (2) negative control group (empty eukaryotic expression vector), (3) positive control (GAPDH), (4) Marker, and (5-12) TNFRSF6B (1-8 transformants). (F) Western blot analysis of three different groups including control group (CON), which is not transfected with any plasmid in Capan-1 cells (empty cells in control group), NC was transfected with expression plasmid and negative control cell group was transfected with viral vector plasmid (negative control group). $\mathrm{KD} 1,2,3$, 4 contain the target genes for the different interference target sequences of RNAi viral vector plasmid serial number. The corresponding interference plasmid serial were KD1 (4209), KD2 (4210), KD3 (4211), and KD4 (4212). The data shown are representative of three independent experiments with the similar results.

the growth of pancreatic cancer Capan-1 cells by quantifying the viable cells after treating cells with lentivirusmediated shRNA targeting DCR-3 for various time periods using MTT assay. The results reveal that lentivirus-mediated shRNA targeting DCR-3 inhibited the growth of pancreatic cancer Capan-1 cells in a timedependent manner (Figure 4A).
Apoptosis, autophagy, and necrosis are the major types of cell death (Leist and Jaattela, 2001). Among the three major pathways of cell death, apoptosis is the most well planned and orderly mode of cell death (Elmore, 2007; Hengartner, 2000). More than $50 \%$ of neoplasms undergo aberrations in the apoptotic machinery which leads to abnormal cell proliferation (Mashima and Tsuruo, 2005; 


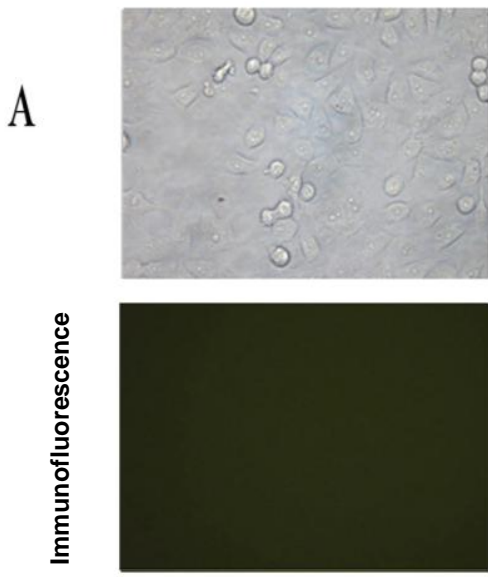

CON
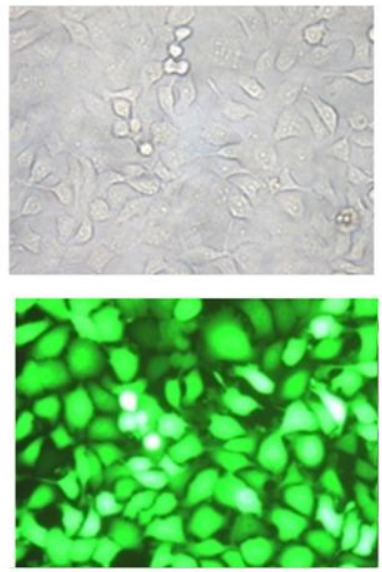

$\mathrm{NC}$
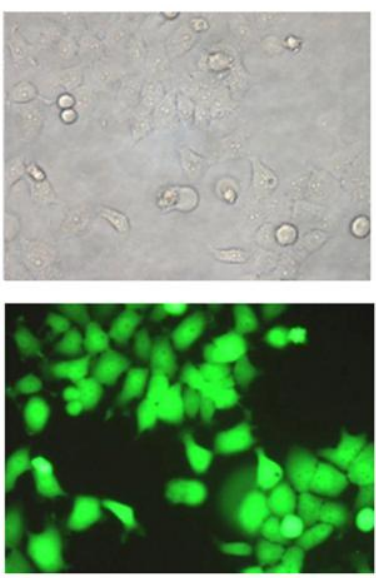

KD

B

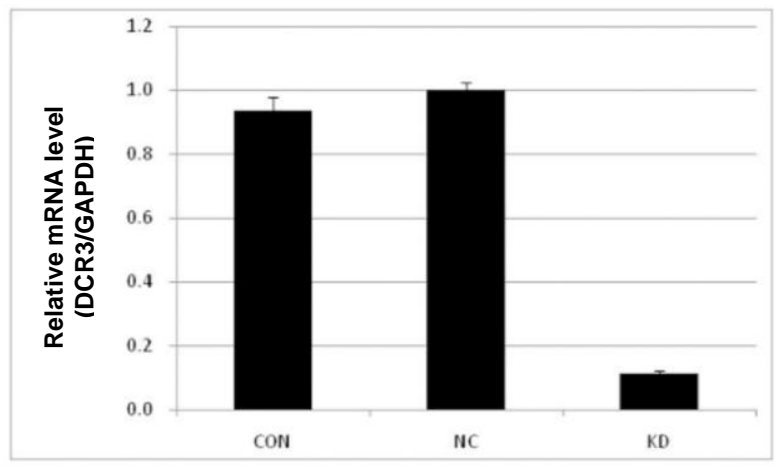

C

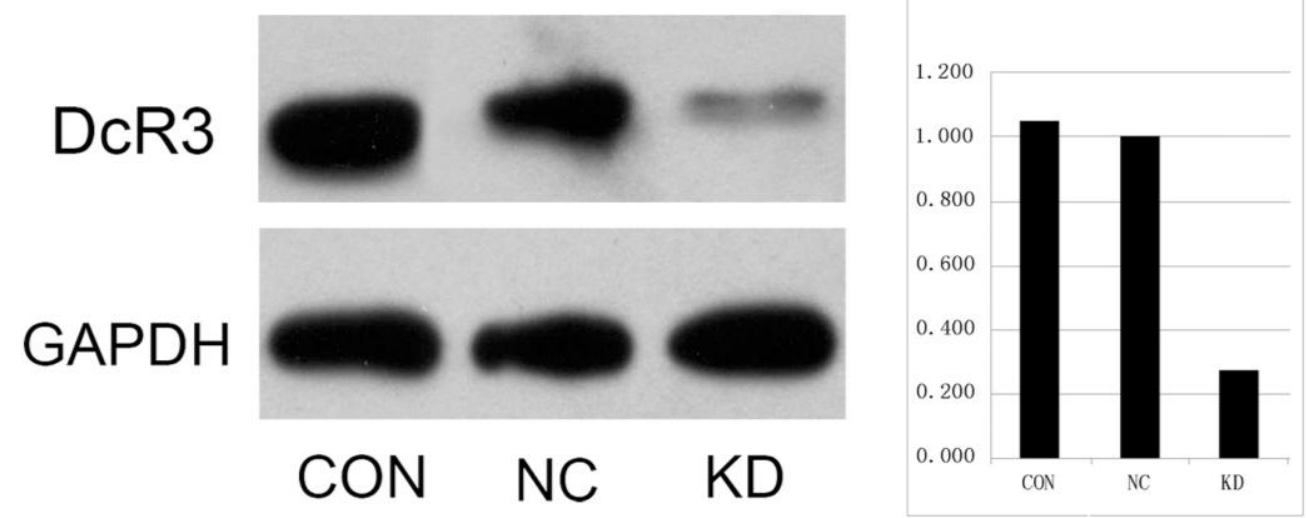

Figure 3. Effects of lentivirus-mediated shRNA targeting DcR-3 on cell morphology and reverse transcriptase-PCR and immunoblotting assessment of decoy receptor 3 (DcR3) mRNA and protein expression in Capan-1 pancreatic cancer cell line: (A) Morphological changes and fluorescence images (200 ×g, 200 magnification bright field image) of Capan-1 pancreatic cancer cells after the transfection with lentivirus-mediated shRNA (KD) as compared to the control group (CON). (B) Equal amounts of concentrated media from cells were subjected for reverse transcriptase-PCR with the DcR3 primer, representative graph illustrating the percentage of relative amount of mRNA in different groups. (C) Capan1 cells were exposed to lentivirus-mediated shRNA targeting DcR-3 for specified time intervals. Equal amounts of lysate protein were subjected to gel electrophoresis. Expression levels of DcR-3 were monitored in the control group (CON), NC, and KD (knockdown) groups in Capan-1 cells by Western blot assay. Data are representative of at least two independent experiments with similar results.

Pommier et al., 2004). The regulation of apoptosis is therefore the most important in the treatment of cancer (Fulda, 2010; Lawen, 2003; Reed, 2002). Accumulated evidences indicated that most of the chemotherapeutic agents halt tumor cells proliferation via induction of apoptosis (Saha et al., 2010; Wu et al., 2009; Zhang, 


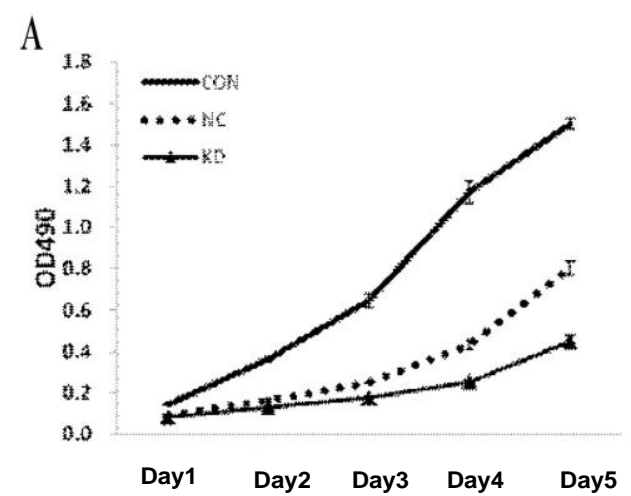

Time

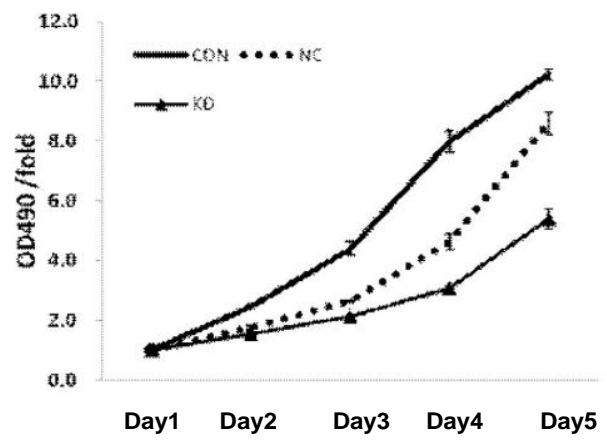

Time

B

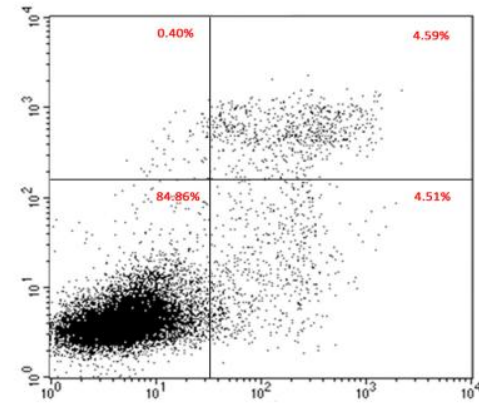

CON

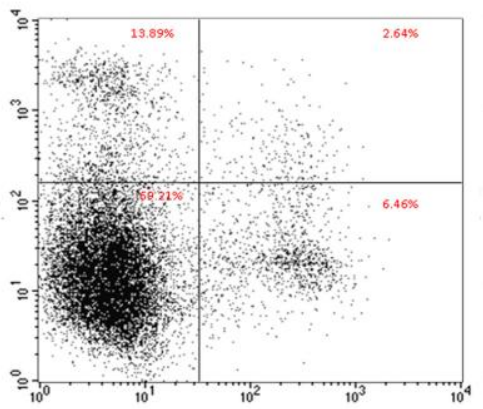

$\mathrm{NC}$

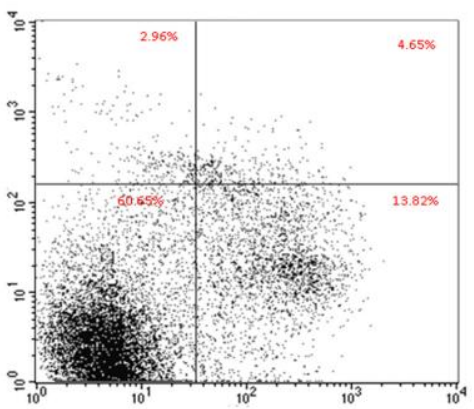

KD

C

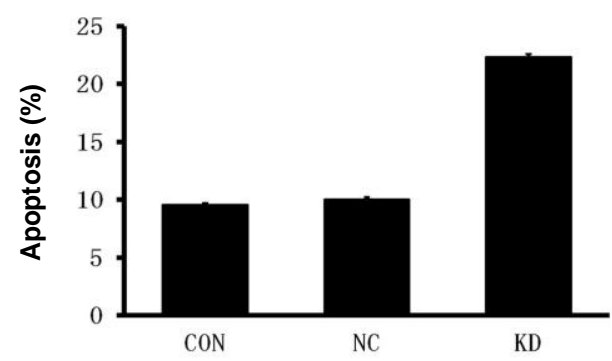

Figure 4. Lentivirus-mediated shRNA targeting DcR-3 inhibits pancreatic cancer Capan-1 cell proliferation and induces apoptosis: (A) Cell viability was determined by MTT assay. The effect of lentivirus-mediated shRNA on the cell growth inhibition of Capan-1 cells was compared with control group. The graph was plotted against mean values of percentages of three independent experiments. (B) Apoptosis induced by lentivirusmediated shRNA targeting DCR-3 in pancreatic cancer Capan-1 cells. Capan-1 cells were treated with lentivirus-mediated shRNA. Then cells were stained with FITC-conjugated Annexin $\mathrm{V}$ and $\mathrm{PI}$ for flow cytometric analysis. The flow cytometry profile represents Annexin V-FITC staining in $x$ axis and PI in $y$ axis. The number represents the percentages of apoptotic cells in each condition. (C) Representative graph illustrating the percentage of apoptotic cells. Statistically significant changes were compared with control group. The data shown are representative of three independent experiments with the similar results.

2002). Here in, we examined whether lentivirus-mediated shRNA targeting DCR-3 inhibited cell growth of pancreatic cancer Capan-1 cells through the induction of apoptosis. Lentivirus-mediated shRNA-induced apoptosis was determined by flow cytometric analysis. The results of flow cytometric analysis showed that the rates of apoptosis were 28.87 and $8.43 \%$ in the cells treated with lenti-sh-RNA-DcR3 or lenti-NC, respectively as compared to the $7.31 \%$ in control cells (Figure 4B and C). Our results demonstrated that shRNA decreased DcR3 expression and enhances apoptosis in human pancreatic cancer Capan-1 cells. 
Taken together, our results suggest that the shRNAmediated decrease in the levels of DcR3 significantly inhibited cell proliferation and enhanced apoptosis in human pancreatic cancer cells. These results suggest that lentivirus-mediated gene therapy targeting DcR3 is a potential and attractive strategy for the treatment of pancreatic cancer.

\section{REFERENCES}

Awasthi N, Kirane A, Schwarz MA, Toombs JE, Brekken RA, Schwarz RE (2011). Smac mimetic-derived augmentation of chemotherapeutic response in experimental pancreatic cancer. BMC Cancer 11:15.

Elmore S (2007). Apoptosis: a review of programmed cell death. Toxicol. Pathol. 35: 495-516.

Elnemr A, Ohta T, Yachie A, Kayahara M, Kitagawa H, Fujimura T, Ninomiya I, Fushida S, Nishimura GI, Shimizu K, Miwa K (2001). Human pancreatic cancer cells disable function of Fas receptors at several levels in Fas signal transduction pathway. Int. J. Oncol. 18:311-316.

Fish RJ, Kruithof EK (2004). Short-term cytotoxic effects and long-term instability of RNAi delivered using lentiviral vectors. BMC Mol. Biol. 5:9.

Fulda S (2010). Evasion of apoptosis as a cellular stress response in cancer. Int. J. Cell Biol. 2010:370835.

Hengartner MO (2000). The biochemistry of apoptosis. Nature, 407: 770-776.

Jemal A, Siegel R, Ward E, Hao Y, Xu J, Thun MJ (2009). Cancer statistics, 2009. CA Cancer J. Clin. 59: 225-249.

Lawen A (2003). Apoptosis-an introduction. Bioessays 25: 888-896.

Leist M, Jaattela M (2001). Four deaths and a funeral: from caspases to alternative mechanisms. Nat. Rev. Mol. Cell. Biol. 2: 589-598.

Li W, Zhang C, Chen C, Zhuang G (2007). Correlation between expression of DcR3 on tumor cells and sensitivity to FasL. Cell Mol. Immunol. 4: 455-460.

Mashima T, Tsuruo T (2005). Defects of the apoptotic pathway as therapeutic target against cancer. Drug Resist. Updat. 8: 339-343.

Pitti RM, Marsters SA, Lawrence DA, Roy M, Kischkel FC, Dowd P, Huang A, Donahue CJ, Sherwood SW, Baldwin DT, Godowski PJ, Wood WI, Gurney AL, Hillan KJ, Cohen RL, Goddard AD, Botstein D, Ashkenazi A (1998). Genomic amplification of a decoy receptor for Fas ligand in lung and colon cancer. Nature 396:699-703.

Pommier Y, Sordet O, Antony S, Hayward RL, Kohn KW (2004). Apoptosis defects and chemotherapy resistance: molecular interaction maps and networks. Oncogene 23:2934-2949.

Rasul A, Ding C, Li X, Khan M, Yi F, Ali M, Ma T (2012). Dracorhodin perchlorate inhibits $\mathrm{PI} 3 \mathrm{~K} / \mathrm{Akt}$ and NF-kappaB activation, up-regulates the expression of p53, and enhances apoptosis. Apoptosis DOI: 17:1104-1119.

Rasul A, Khan M, Yu B, Ma T, Yang H (2011). Xanthoxyletin, a Coumarin Induces S Phase Arrest and Apoptosis in Human Gastric Adenocarcinoma SGC-7901 Cells. Asian Pac. J. Cancer Prev. 12:1219-1223.
Reed JC (2002). Apoptosis-based therapies. Nat. Rev. Drug Discov., 1: 111-121.

Saha A, Kuzuhara T, Echigo N, Suganuma M, Fujiki H (2010). New role of (-)-epicatechin in enhancing the induction of growth inhibition and apoptosis in human lung cancer cells by curcumin. Cancer Prev. Res. Phila 3:953-962.

Shawi AA, Rasul A, Khan M, lqbal F, Ma T (2011). Eupatilin: A flavonoid compound isolated from the artemisia plant, induces apoptosis and G2/M phase cell cycle arrest in human melanoma A375 cells. Afr. J. Pharm. Pharmacol. 5: 582-588.

Shen HW, Gao SL, Wu YL, Peng SY (2005). Overexpression of decoy receptor 3 in hepatocellular carcinoma and its association with resistance to Fas ligand-mediated apoptosis. World J. Gastroenterol. 11: 5926-5930.

Thomas M, Greil J, Heidenreich O (2006). Targeting leukemic fusion proteins with small interfering RNAs: recent advances and therapeutic potentials. Acta Pharmacol. Sin. 27:273-281.

Wang H, Tan SS, Wang XY, Liu DH, Yu CS, Bai ZL, He DL, Zhao J (2007). Silencing livin gene by siRNA leads to apoptosis induction, cell cycle arrest, and proliferation inhibition in malignant melanoma LiBr cells. Acta Pharmacol. Sin. 28:1968-1974.

Wu XJ, Hu Y, Lamy E, Mersch-Sundermann V (2009). Apoptosis induction in human lung adenocarcinoma cells by oil-soluble allyl sulfides: triggers, pathways, and modulators. Environ. Mol. Mutagen. 50:266-275.

Wu Y, Han B, Sheng H, Lin M, Moore PA, Zhang J, Wu J (2003). Clinical significance of detecting elevated serum DcR3/TR6/M68 in malignant tumor patients. Int. J. Cancer 105:724-732.

Yip-Schneider MT, Nakshatri H, Sweeney CJ, Marshall MS, Wiebke EA, Schmidt CM (2005). Parthenolide and sulindac cooperate to mediate growth suppression and inhibit the nuclear factor-kappa B pathway in pancreatic carcinoma cells. Mol. Cancer Ther. 4:587-594.

Zhang JY (2002). Apoptosis-based anticancer drugs. Nat. Rev. Drug Discov. 1:101-102.

Zhang L, Liu HJ, Li TJ, Yang Y, Guo XL, Wu MC, Rui YC, Wei LX (2008). Lentiviral vector-mediated siRNA knockdown of SR-PSOX inhibits foam cell formation in vitro. Acta Pharmacol. Sin. 29:847-852. 